BERNARD FROCHOT, VINCENT GODREAU

les objectifs des opérations menées en vue d'y redonner une place à la flore et à la faune.

\section{DIVERSITÉ DES BIOTOPES}

Considérés comme supports de développement de nouveaux écosystèmes, les sites peuvent présenter des conditions abiotiques très diverses, selon la nature du substrat et les modalités d'extraction des roches et de remise en état des lieux. Essayons de classer ces différents biotopes.

\section{Selon les conditions géologiques}

La texture du sous-sol nous amène à ranger les sites miniers en trois principales catégories :

\section{Carrières et mines en roche massive}

La nature géologique de la roche et la "morphologie" de l'excavation (à faible relief ou bien présentant pentes et falaises artificielles, etc.) vont être des facteurs écologiques déterminants, aussi bien pour les communautés végétales que pour les espèces animales. Ces milieux massifs sont le plus souvent secs, ou bien peuvent dans certains cas abriter des collections d'eau, le plus souvent des mares.

\title{
RÉSUMÉ : Intérêt écologique des carrières, terrils et mines
}

Les sites d'extraction de roche sont colonisés spontanément ou non par la faune et la flore. II s'y développe des écosystèmes nouveaux caractérisés par une faible productivité biologique, des conditions de vie originales, l'isolement et aussi une pression humaine réduite.

Leurs peuplements de plantes et d'animaux sont souvent originaux avec des espèces rares pour la région. Ces écosystèmes évoluent rapidement au cours des années qui suivent l'exploitation, selon des successions écologiques.

Le suivi pendant vingt ans de l'évolution du peuplement d'oiseaux de carrières en eau en Bourgogne donne un exemple de telles successions. Après abandon de l'exploitation, la liste des espèces, l'abondance, la diversité, etc., changent rapidement d'année en année. Mesuré selon les critères du nombre et de la rareté des espèces, l'intérêt écologique du site étudié évolue en parallèle : il augmente rapidement, puis culmine vers la septième année, et décroît ensuite progressivement.

La banalisation du peuplement d'oiseaux observé au terme de ces vingt années correspond-elle à une tendance générale ? Cette hypothèse mérite d'être étudiée pour d'autres taxons et d'autres types de sites. Une synthèse des études actuellement disponible sur les écosystèmes de carrière fait apparaitre des besoins en matière de recherche : il faudrait en particulier des suivis de leur évolution et élaborer des méthodologies permettant d'évaluer précisément leur intérêt écologique.
Résumé en anglais p. 76

\section{Carrières et mines en roche meuble}

Il faut de toute évidence distinguer les carrières sèches des carrières en eau. Les premières se trouvent surtout dans les terrasses alluvionnaires latérales aux cours d'eau ou délaissées par ceux-ci, et plus localement dans des sables fossiles, des éboulis ou gisements de scories volcaniques. Ce sont des biotopes particulièrement arides, qui peuvent différer par la granulométrie du substrat, la profondeur de l'excavation, la pente des fronts de taille, etc.

Les carrières en eau, maintenant nombreuses, sont des excavations atteignant la nappe phréatique où s'installent des écosystèmes d'eau permanente. La nature chimique et granulométrique du substrat, la qualité de l'eau de nappe et la morphologie des bassins y sont des facteurs écologiques de première importance, ainsi que l'amplitude des variations du niveau d'eau, qui peut être limitante pour diverses espèces de plantes ou d'oiseaux (Bournaud et al., 1980). Lorsque ces dernières entraînent l'assèchement saisonnier du plan d'eau, on y trouve toutefois des communautés animales et végétales particulières, adaptées à vivre en eau temporaire (Richardot, 1983).

\section{Terrils}

Il s'agit là de substrats de roche meuble, déplacée et remodelée, donc de biotopes extrêmement artificiels. Il s'y développe pourtant des biocénoses originales et même caractéristiques de ce type d'habitat (Petit, 1983). 


\section{"CARRIÈRES ET MINES "}

Le code minier distingue carrières et mines, en leur attribuant des régimes juridiques bien différents : les carrières sont les lieux d'extraction des roches les plus courantes, dont sont tirés principalement les matériaux de construction et viabilité, tandis que les mines sont, par définition, les extractions de roches plus rares et précieuses (minerais, roches énergétiques, certains sels, ...). Les matériaux extraits des carrières restent à disposition du propriétaire du sol, pas ceux des mines...

Ce sont les mines qui poseront le plus souvent des problèmes de pollution du sol et des eaux (notamment métalliques). Les carrières, moins polluantes, couvrent par contre des surfaces considérables: I'emprise territoriale de l'ensemble des carrières de notre pays était estimée à $1200 \mathrm{~km}^{2}$ en 1983 (Conseil Général des Mines, 1983) avec une progression annuelle de $50 \mathrm{~km}^{2}$.

Les carrières en roche meuble sont le plus souvent creusées dans les sables et graviers alluviaux (d'où leur appellation fréquente de "sablières" ou "gravières"), plus rarement dans des éboulis, des dépôts de pouzzolanes, ... Elles représentent une part majoritaire des extractions de roches, en raison de l'importance de la demande en "granulats". Ces derniers proviennent également, et de plus en plus, du concassage de roches massives.

Source : Conseil Général des Mines (1983), Politiques locales des carrières, Paris.

\section{CARRIERES (ou MINES) EN ROCHE MASSIVE}

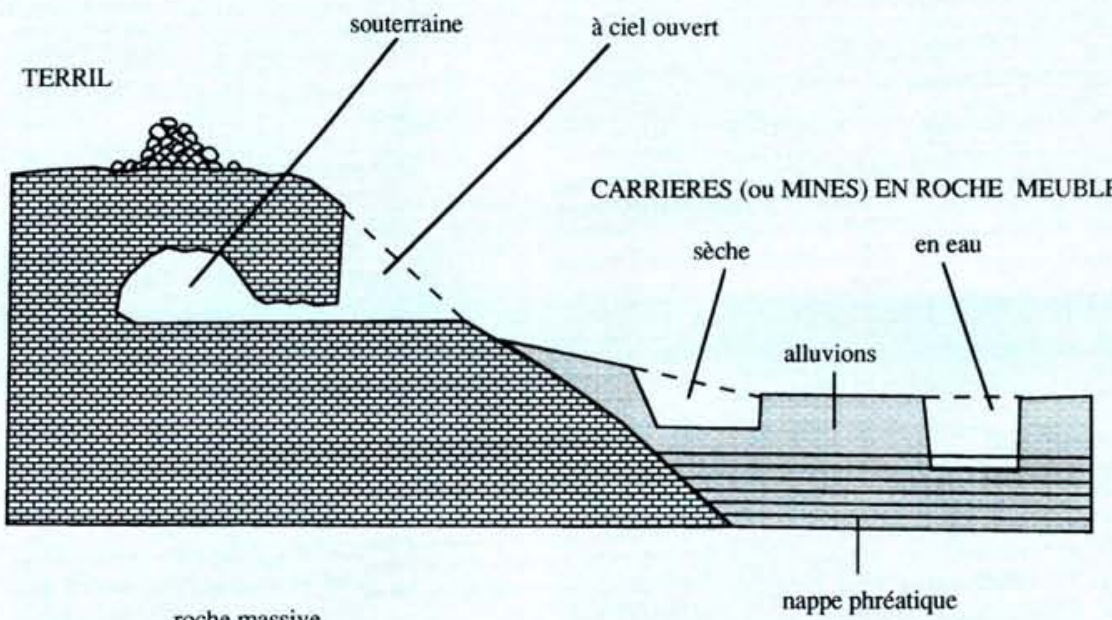

roche massive

nappe phréatique 


\section{Selon les conditions édaphiques}

Le sol, nécessairement décapé dans le cas des exploitations à ciel ouvert, peut être parfois restitué au site après la fin des travaux, ce qui aura d'importantes répercussions écologiques et nous amène à distinguer nettement deux ensembles de milieux.

\section{Biotopes à sol restitué}

Certains gisements alluvionnaires à sec permettent la réinstallation du sol après enlèvement d'une certaine épaisseur de substrat rocheux ou de granulats, et la réinstallation d'un écosystème voisin de l'écosystème initial. Ce procédé a permis un certain nombre de "remises en état agricole", qui semblent maintenant moins opportunes en période de déprise ; il autorise aussi divers types de boisements. Malgré ces réussites, les cas de remise en place du sol restent assez rares ; les milieux qui en résultent retrouvent une certaine productivité mais perdent, notamment pour cette raison, beaucoup d'originalité écologique : nous n'en tiendrons pas compte dans la suite du texte.

\section{Biotopes à sol exporté}

Le cas est fréquent pour les carrières sèches (et d'une manière générale pour les exploitations profondes et prolongées) et bien entendu de règle pour les carrières en eau, permanentes ou temporaires. Le biotope restitué à l'environnement après les travaux se trouve alors presque purement minéral, et dépourvu des traces de son passé biologique : on peut le considérer comme un substrat permettant le développement d'une succession écologique primaire.

\section{CARACTÈRES ÉCOLOGIQUES GÉNÉRAUX DES SITES MINIERS}

Considéré en termes de productivité biologique et de biodiversité, l'intérêt écologique des sites de mines et carrières présente des traits caractéristiques, dont nous soulignons ci-dessous les principaux.

\section{Faible productivité}

Les sites d'extraction ou de déblai se caractérisent le plus souvent par une productivité biologique faible ou très faible, en raison de la rareté de la matière organique ou des nutriments dans le substrat, qui reste essentiellement minéral après l'exportation du sol. Ce caractère semble valoir aussi bien pour les substrats de roche mère compacte que pour ceux de roche meuble, fractionnée en blocs, graviers ou limons. Le $\mathrm{pH}$ est bien entendu aussi un facteur important et la richesse en espèces particulièrement basse en site acide (Grunwald et al., 1995).

Si le site est alimenté en eau, il s'agit le plus souvent de l'eau phréatique, dont la charge nutritive est normalement faible. Le milieu ainsi constitué reste souvent à un niveau de productivité oligo- ou mésotrophe (Moreau, 1982 ; Cardinal, 1983 ; Garnier et al., 1987 ; Brunaud et al., 1988 ; Gambade et Simonin-Guengard, 1991). Les carrières alimentées par de l'eau enrichie en nutriments, d'origine agricole ou autre, et plus particulièrement les bassins connectés aux grands cours d'eau, peuvent présenter un niveau trophique bien supérieur, parfois même eutrophe (Garnier, 1982 ; Moreau, 1982 ; Testard, 1983 ; Vermorel, 1985 ; Garnier et al., 1987).

\section{Pression humaine réduite}

Cette faible productivité naturelle, associée à de fréquentes difficultés d'exploitation, semble jusqu'à présent empêcher toute exploitation intensive des sites miniers : les sites secs restent épargnés par l'agriculture et même les carrières en eau ne semblent pas permettre une pisciculture de production rentable. En dehors des cas où le site est urbanisé ou bien - de moins en moins souvent - sert de réceptacle à des déchets divers, la principale réutilisation reste l'accueil d'activités de loisirs, sauvages ou organisés ; même si cette fréquentation peut présenter des risques pour l'environnement, elle laisse place, le plus souvent, au développement spontané de nombreuses espèces végétales et animales. C'est certainement pour cette raison essentielle que les carrières et mines abandonnées acquièrent rapidement une valeur écologique plus forte que les milieux qu'elles ont remplacés ou qui les entourent.

\section{Originalité}

Qu'ils soient secs ou humides, les sites d'extraction sont colonisés par des biocénoses originales (Lefeuvre et Le Duc, 1981 ; G.R.M.R.B., 1983 ; Petit, 1983 ; Carteron et Cachot, 1985 ; Arnal et Lamade, 1987 ; Robert et al., 1991). Par leurs caractères abiotiques et leur composition floristique et faunistique, ces écosystèmes neufs se rapprochent plus ou moins de ceux des falaises ou éboulis naturels, ou encore des bancs de graviers, falaises sableuses ou bras morts issus de la dynamique naturelle des cours d'eau. Ces milieux naturels sont d'ailleurs le réservoir des espèces qui colonisent les carrières. D'où l'originalité des écosystèmes miniers, qui attirent dans un environnement souvent banal des espèces inféodées aux falaises, grandes rivières, dunes... et autres milieux rares ou bien déjà très abîmés par l'homme'.

\section{Importance de l'âge du milieu}

La colonisation par des espèces pionnières peut être très rapide, notamment en site humide (souvent dès le début des travaux d'extraction, Desbrosses, 1982), bien entendu par des espèces animales mobiles, mais aussi par des plantes supérieures, des mollusques (Genin, 1987), etc. Les écosystèmes ainsi constitués évoluent ensuite (Glue, 1970 ; Milne, 1974 ; Frochot et Renier, 1984 ; Jefferson et Usher, 1986, Arnal et Lamade, 1987), d'abord rapide. ment, puis avec la décélération observée classiquement au long des successions écologiques, avec transformation parallèle de la biocénose (en particulier, sa composition spécifique se modifie) et du biotope (par érosion, changement de la qualité de l'eau....). Au cours de cette évolution naturelle, les espèces pionnières disparaissent au profit de celles inféodées aux stades successionnels plus mûrs. À titre d'exemple, les carrières de 
granulats alluvionnaires sont colonisées par des espèces (comme le Petit gravelot Charadrius dubius) habitant normalement les dépôts sableux neufs des rivières : l'entreprise d'extraction de granulats joue sur l'écosystème le rôle d'une perturbation analogue aux crues des cours d'eau. Mais à l'échelle d'une seule carrière la comparaison s'arrête là : les espèces pionnières ne se maintiennent en rivière que dans la mesure où la dynamique fluviale y entretient régulièrement un régime de perturbations rajeunissantes, tandis qu'elles ne peuvent subsister en carrière une fois que les effets de l'extraction sont estompés (Amoros et Petts, 1993).

D'une manière plus générale, on doit donc s'attendre à une évolution de la valeur écologique des sites de carrières, réaménagés ou non, au fil des années. La disparition des espèces pionnières, souvent plus originales, va-t-elle toujours amoindrir l'intérêt écologique du site? L'évolution de la succession vers les stades mûrs, à biocénoses plus banales (même si elles sont parfois plus productives ou à cause de cela) est-elle inéluctable? La réponse à ces questions nécessite certainement encore des études de terrain, et notamment des suivis à long terme ; nous l'abordons ci-dessous à partir d'un exemple (cf. paragraphe "Successions écologiques").

\section{Isolement}

Les populations de plantes et d'animaux habitant les carrières sont très souvent en situation d'isolement géographique, surtout lorsque l'extraction a créé un site original par rapport à son environnement : gravière en eau isolée en région sèche, carrière en roche massive éloignée de toute falaise naturelle, etc. De plus, les sites d'extraction sont souvent relativement petits (leur superficie reste souvent de l'ordre de la dizaine d'hectares, ce qui est très faible pour les espèces exigeantes en espace, dont beaucoup de Vertébrés). Pour ces deux raisons, les écosystèmes des carrières mérite. raient d'être étudiés aussi à la lumière des diverses théories de l'insularité, aussi bien pour comprendre leur fonctionnement que pour organiser leur gestion éventuelle (Simberloff, 1986).

\section{Impacts sur le sol et l'eau}

À l'influence directe des carrières sur la flore et la faune, s'ajoutent leurs effets sur le biotope. Ceux-ci peuvent être négatifs, dans le cas des minerais toxiques ; certaines mines de Cuivre, de Zinc,... polluent non seulement le site d'extraction mais aussi les eaux de circulation. La végétalisation du site, avec des espèces robustes et persistantes, peut être un moyen de contenir les éléments polluants et de les neutraliser (Brett, 1994 ; Jaffre et al., 1994).

Des expériences de végétalisation de sites d'exploitation du pétrole en Alaska se sont avérées nécessaires pour décontaminer des zones ayant subi des déversements de pétrole, d'eau de mer et de boue de forage (Jorgenson et Joyce, 1994).

Inversement, beaucoup de carrières abandonnées ont globalement sur la qualité du sol et de l'eau des effets plutôt positifs, dans la mesure où ces sites deviennent libérés de toute exploitation intensive. Ce sont des espaces où les sols se reconstituent (Moiroud et Capellano, 1994 ; Spain et Lavelle, 1994) et où les eaux de circulation trouvent de bonnes conditions de qualité. Plusieurs études ont ainsi montré un effet épurateur des carrières en eau sur les nappes phréatiques, jouant au moins à l'encontre des nitrates (Eberentz et Rinck, 1987 ; Labroue et al., 1988). Ces effets des carrières sur les nappes sont en fait relativement nombreux et complexes (Peaudecerf, 1975, 1977) : ils concernent les niveaux phréatiques, la température de l'eau, son évaporation... Souvent négligeables pour une exploitation isolée et petite, ils peuvent se cumuler et perturber le cycle de l'eau quand les carrières couvrent de grandes étendues. Le colmatage des berges, qui s'accroît lors du vieillissement des sablières en eau, ou qui provient des modalités d'extraction ou de remise en état des lieux, peut réduire beaucoup les échanges gravière - nappe, et par là influencer le fonctionnement écologique des plans d'eau, dans un sens généralement défavorable à leur
1. A titre d'exemple, les carrières en eau de Quincey (Bourgogne : cf. infra) ont éte colonisées par des especes aviennes aussi rares que le Butor blongios (lxobrychus minutus) ou la Locustelle luscinioide (Locustella luscinioides) et par des plantes classées rares à l'échelle régionale ou nationale (dont Epipactis palustris).

En carrières sèches, Robert et al. démontrent l'intérêt des anciennes exploitations en tant que réservoir d'espèces d'insectes exceptionnelles en Franche-Comté, notamment du groupe des Hyménoptères aculéates (G.R.M.R.B., 1983). 
intérêt biologique. Ce facteur mérite d'être pris en compte lors de l'exploitation, de la réaffectation et du suivi des carrières en eau (Durbec, 1986).

\section{RECRÉER LA NATURE ?}

Les actions, maintenant fréquentes, visant à redonner une place à la nature dans les carrières abandonnées, sont très diverses, aussi bien dans leurs modalités que dans leurs objectifs. Leurs auteurs les décrivent sous une terminologie hétéroclite, allant du "reverdissement" à la "création d'écosystèmes", qui traduit peut-être la difficulté de choisir des références et de les bien choisir.

Avant de discuter ces points, un constat s'impose : en l'absence de toute intervention humaine, la colonisation strictement naturelle des carrières abandonnées est rapide et importante, avec constitution d'écosystèmes neufs dont nous avons souligné ci-dessus les caractères. Les actions entreprises en faveur de la valeur écologique de ces sites ne justifient donc pas complètement de l'appellation "création d'écosystèmes" : elles se limitent à orienter ce phénomène naturel, le plus souvent en favorisant tel ou tel type d'habitat, donc tel ou tel groupe d'espèces.

\section{Terminologie}

Seules les carrières dont le sol est restitué peuvent retrouver rapidement, après la remise en état des lieux, des écosystèmes proches de ceux de l'état initial (dans la mesure où le sol n'a pas été trop modifié par les travaux). Ce traitement relève donc d'un processus de restauration du milieu naturel (Aronson et al., 1993, Le Floc'h et Aronson, 1994).

Dans les nombreux cas où le sol a été exporté, le nouvel écosystème, sec ou humide, est très différent de l'état antérieur, et souvent complètement nouveau. Pour cette raison, les actions menées en sa faveur peuvent difficilement être dénommées réhabilitations, mais entrent plutôt dans le cadre de la réaffectation selon Le Floc'h et Aronson (en anglais reallocation selon Aronson et al., 1993).

\section{Écosystèmes de référence}

Puisque les écosystèmes de carrières sont très largement artificiels, leur gestion doit se faire en l'absence d'une référence naturelle parfaite. On peut cependant trouver des modèles parfois assez proches parmi les écosystèmes dont semblent provenir les espèces colonisatrices de la carrière, et qui présentent des conditions abiotiques voisines : falaises pour les fronts de taille en roche massive, éboulis pour les terrils, grandes rivières libres pour les sablières en eau (Tester, 1992). On peut aussi se référer à des carrières plus anciennes, dont l'état écologique a été suivi.

Soulignons aussi qu'il pourrait être très utile de disposer de références pour l'évaluation écologique des sites et des interventions humaines. En tout état de cause, ces dernières ne devraient pas se limiter à des inventaires biologiques bruts, mais devraient se référer à l'état prévisible du système en l'absence d'intervention : il faut bien faire la part de ce qui revient d'un côté au gestionnaire de l'espace et de l'autre à la nature, puisque l'écosystème finalement restitué résulte de leur double paternité. De telles prévisions ne peuvent guère s'appuyer que sur des cas antérieurs connus et très étudiés.

\section{SUCCESSIONS ÉCOLOGIQUES}

L'importance et la rapidité des transformations que subit l'écosystème nouveau dès l'abandon du site de carrière sont telles que la gestion doit les prendre en compte impérativement. On ne connaît aujourd'hui que les grandes tendances de ces successions écologiques (Luken, 1990) et des recherches approfondies sont souhaitables. Nous l'illustrerons par un exemple.

\section{Un cas de suivi diachronique: les sablières de Quincey}

En 1973, notre équipe participe au réaménagement "écologique" de sablières en eau à Quincey, quelque $20 \mathrm{~km}$ au sud de
Dijon (Côte-d'Or), au cœur d'une vaste plaine agricole parsemée de petits étangs forestiers et en bordure de la côte viticole (Frochot et Renier, 1984).

Ce site est un ensemble de quatre grandes sablières provenant de l'exploitation de matériaux nécessaires à la construction de l'autoroute reliant Dijon à Beaune. Les travaux se déroulent en 1972, et s'interrompent simultanément sur les quatre sites en 1974.

L'extraction des graviers abandonne un ensemble de 4 bassins proches, d'une superficie totale en eau d'environ 40 hectares. En vue d'une réutilisation pour les loisirs (planche à voile, camping, moto-cross, pêche) et d'une intégration paysagère, chaque bassin est réaménagé avec des berges en pente très faible sur une partie de son pourtour et quelques hauts fonds mais aucun ensemencement ni apport de sol n'est réalisé. Aucun radeau favorable à la nidification de certaines espèces d'oiseaux (Sternes principalement) n'a été installé ; seuls sont introduits des Poissons en 1976.

La colonisation spontanée du milieu par la végétation est très rapide : les ceintures de massettes à Typha latifolia et T. angustifolia s'installent dès 1975 et le développement de la phragmitaie à Phragmites aus. tralis devient important à partir de 1978. Une faune variée d'Invertébrés et d'Oiseaux occupe le site dès la fin de l'extraction et la mise en eau. Pour la plupart des espèces colonisatrices, les zones sources assurant la recolonisation sont situées dans un rayon de quelques kilomètres autour du site.

De la fin des travaux, en 1974, à 1994, le site n'a pas été sensiblement modifié, soumis seulement à une pression constante de pêcheurs et baigneurs, de sorte que l'évolution des écosystèmes aquatiques relève surtout du processus naturel d'une succession écologique. Nous avons observé cette évolution pendant cette période d'environ vingt années, et plus particulièrement réalisé chaque année, à partir de 1975 , le dénombrement du peuplement des oiseaux aquatiques nicheurs, par la méthode standardisée de cartographie des territoires. Nous 


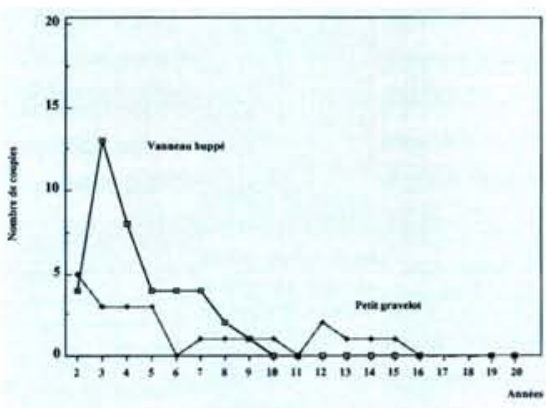

Figure 1 - Evolution du nombre de couples nicheurs de Vanneau huppé (Vanellus vanellus) et de Petit gravelot (Charadrius dubius) en fonction de l'áge de la sablière (en années).

présentons ci-après les principales informations apportées par cette recherche.

\section{Succession des espèces}

Les oiseaux ont très rapidement adopté les nouveaux plans d'eau comme site de nidification. Le suivi de leurs peuplements montre un processus conforme aux théories générales des successions écologiques (Clements, 1916 ; Odum, 1969 ; Blondel, 1976, 1979 ; Lepart et Escarre, 1983 ; Odum, 1993), en opposant, en particulier, des espèces pionnières à d'autres inféodées aux stades mûrs. Les premières atteignent des densités maximales dans les toutes premières années de vie du nouvel écosystème. Ce sont surtout le Vanneau huppé Vanellus vanellus et le Petit gravelot Charadrius dubius qui trouvent sur le pourtour des bassins des conditions édaphiques voisines de celles des grèves des cours d'eau ; ces espèces disparaissent en quelques années, quand la végétation réussit à coloniser ce substrat de graviers (figure 1). Le cas du Grèbe castagneux Tachybaptus ruficollis est plus difficile à expliquer : nos recensements (confirmés par des observations sur d'autres carrières en eau) montrent que cette espèce est une pionnière caractéristique, dont la densité chute brutalement dès la deuxième année (figure 2) jusqu'à extinction. En Grande Bretagne, Hughes (1992) observe un taux de reproduction plus fort sur les sites neufs. Les causes de cette évolution d'un oiseau par ailleurs très commun dans beaucoup de milieux humides restent inconnues

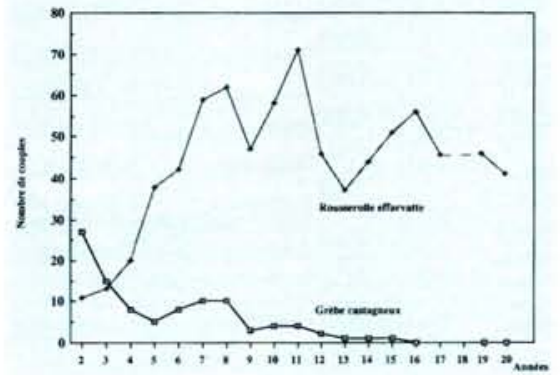

Figure 2-Evvolution du nombre de couples nicheurs de Grèbe castagneux (Tachybaptus ruficollis) et de Rousserolle effarvatte (Acrocephalus scirpaceus) en fonction de l'àge de la sablière (en années)

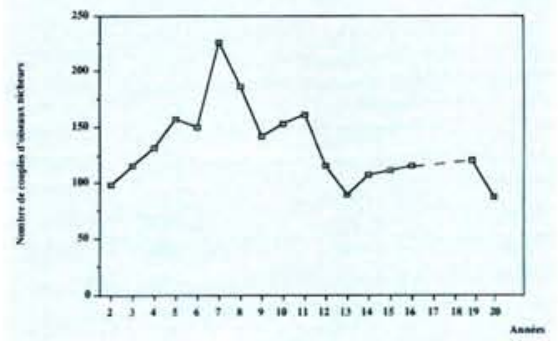

Figure 3 - Évolution de l'abondance (nombre de couples d'oiseaux nicheurs en fonction de l'áge de la sablière (en années).

et justifieraient d'une étude complète des écosystèmes aquatiques concernés (hypothèses : compétition avec d'autres espèces ? prédation par le Brochet Esox lucius (Milne, 1974) ?). Parmi les espèces habitant les stades plus mûrs de la succession (dans les limites de notre fenêtre d'observation de vingt années), citons le Grèbe huppé Podiceps cristatus, les Rallidés, les Rousserolles Acrocephalus scirpaceus et A. arundinaceus (figure 2).

\section{Évolution générale du peuplement d'oiseaux}

Diverses analyses de l'ensemble des espèces nicheuses font apparaître des tendances successionnelles globales.

L'abondance totale du peuplement (figure 3) augmente assez rapidement au début de la succession, culmine au stade de 7 à 8 ans, puis décroît pour se stabiliser à un niveau médiocre. Cette chute provient d'une part de la disparition d'espèces pionnières ou intermédiaires et d'autre part d'une baisse de 


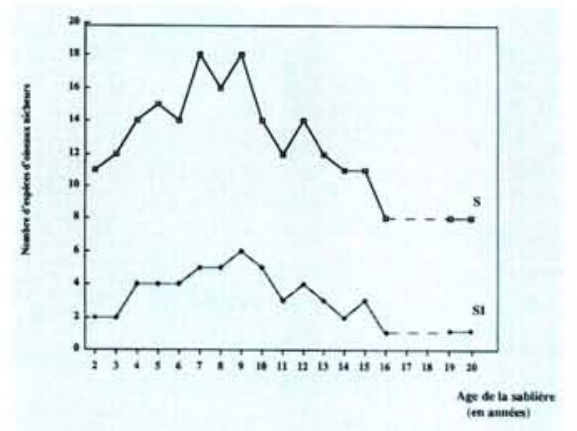

Figure 4 - Evolution de la richesse totale (S) (nombre d'espéces d'oiseaux nicheurs) et de la richesse en especes inscrites (SI) en fonction de l'âge de la sablière (en années) $\mathrm{SI}$ = espéces bénéficiant d'un statut de protection (Liste Rouge et/ou Liste Ramsar France)

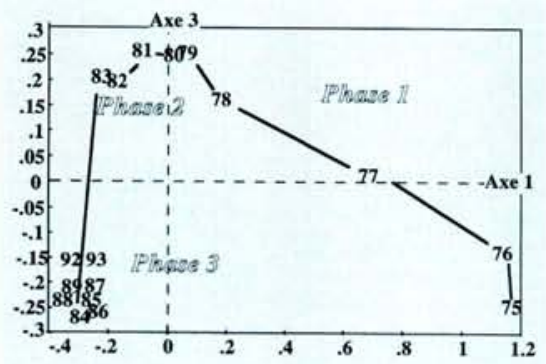

Figure 5 - Analyse factorielle des correspondances (A.F.C.) sur l'ensemble des 17 dénombrements d'avifaune : projection des années selon les axes 1/3.

densité affectant presque toutes les espèces (dont la Foulque Fulica atra, la Poule d'eau Gallinula chloropus, le Grèbe huppé Podiceps cristatus) : elle semble traduire une baisse de la productivité générale du milieu.

La richesse spécifique (figure 4) suit une évolution voisine mais un peu plus contrastée : croissance jusqu'au stade 7 à 9 ans (jusqu'à 18 espèces) puis décroissance jusqu'à un niveau très médiocre (8 espèces seulement en 1989-93). Pour apprécier ces valeurs de la richesse, nous pouvons les comparer à celles mesurées sur un échantillon d'étangs à digue de la même région (Roche, 1982) : nos gravières réaménagées présentent, dans les premières années, des richesses en oiseaux d'eau analogues à celles d'étangs véritables de même dimension, mais nettement plus faibles aux stades 15 . 20 ans. Les richesses très élevées des années
4 à 12 s'expliquent notamment par la coexistence, à ce stade de la succession, d'espèces pionnières pas encore disparues (Petit gravelot Charadrius dubius), d'espèces intermédiaires liées à la végétation d'hélophytes (dont la Locustelle luscinioïde Locustella luscinioides) ou à l'eau (Fuligule milouin Aythia ferina) et des espèces des stades plus mûrs déjà installées (Grèbe huppé Podiceps cristatus, Rousserolles Acrocephalus scirpaceus et $\mathrm{A}$. arundinaceus) : ce mécanisme d'évolution de la richesse est très semblable à celui décrit par Bornette et al. (1994) pour les successions végétales se développant dans les mortes fluviales.

La vitesse de renouvellement évolue au cours des vingt années de suivi, en fonction de la succession des espèces, avec une décélération conforme à celle observée sur des peuplements ornithologiques forestiers (Ferry, 1960 ; Ferry et Frochot, 1970 ; Blondel, 1976, 1979). La position des années sur les axes d'une A.F.C. (figure 5) fait ressortir trois phases successives :

- une phase de colonisation très rapide, de 1975 à 1978, par les espèces pionnières exploitant le terrain nu et les plans d'eau neufs (phase 1) ;

* de 1979 à 1983 une stabilisation à un niveau de richesse spécifique élevé (phase 2) ;

- de 1984 à 1993, une stabilisation à un niveau inférieur très différent, et une évolution du peuplement lente voire nulle les dernières années d'observation (phase 3 ).

Outre cette analyse purement scientifique des caractères collectifs du peuple. ment, on peut s'interroger sur l'évolution de son originalité et de son "intérêt écologique". Une manière, un peu arbitraire mais objective, de l'apprécier peut consister à distinguer, parmi l'ensemble des espèces, celles jouissant d'un statut de protection officiel. lement reconnu au niveau national, européen ou international : il apparaît (figure 4) que ce sont surtout ces espèces classées qui fluctuent et disparaissent progressivement au fil du temps. Selon ce critère, la valeur de l'écosystème en tant qu'habitat de nidifica- tion d'oiseaux varie donc énormément au long des vingt années de la succession.

Tous ces paramètres évoluent en fait en concordance et montrent que l'intérêt ornithologique du site suivi est très important dès l'abandon de l'exploitation des gravières, augmente pour culminer rapidement dans la période entourant la septième année puis décroît pour se stabiliser à une valeur très médiocre au bout de vingt ans (sans préjuger de son évolution future).

L'analyse de la succession végétale montre des liens très probables avec les oiseaux : développement rapide des plantes pionnières, puis des ceintures d'hélophytes qui culminent vers $5-10$ ans puis se stabilisent ou même régressent, en raison de la compétition exercée par la saulaie et probablement aussi d'autres facteurs. Ces observations concordent dans leurs grandes lignes avec celles d'autres auteurs sur la végétation de milieux semblables (Sionneau, 1987, Brunaud et al., 1988) ou en milieux perturbés par l'agriculture puis laissés à l'abandon (Tatoni, 1994). Dans le cas des macrophytes aquatiques ou subaquatiques, le développement de peuplements monospécifiques réduit fortement la diversité floristique des carrières en eau (Cerveaux, 1987).

Remarquons enfin que, malgré tout l'intérêt d'un suivi sur vingt années, ce laps de temps reste bien court comparé à la longévité de l'écosystème étudié ; il sera intéressant de prolonger notre programme d'observations, quitte à augmenter le pas de temps en raison du ralentissement constaté et prévisible des transformations, comme cela se pratique en général pour étudier les successions (Ferry et Frochot, 1970 ; Blondel, 1979).

\section{QUELLE RESTAURATION ?}

\section{Évaluer l'intérêt écologique des carrières}

L'évaluation d'un écosystème reste, dans tous les cas, une opération délicate (Blandin, 1986 ; Brunaud, 1986 ; Usher, 1986b ; Devillers et al., 1990 ; Ledant, 
1991). La façon dont elle est souvent pratiquée pour les sites de carrières et mines nous suggère deux remarques importantes. La première concerne la dimension temporelle : la rapidité des transformations successionnelles dans ces écosystèmes neufs est telle que l'évaluation doit en tenir compte, en se situant à un stade bien précis et daté de la période post-abandon et en envisageant les états futurs prévisibles. La seconde concerne la diversité : la valeur écologique d'un système inclut, certes, sa biodiversité, mais aussi d'autres aspects tels que l'originalité ou la typicité, les impacts sur l'eau ou le sol, etc. Il faut donc éviter la tendance réductrice consistant à ramener la valeur patrimoniale au seul critère de la diversité (Brunaud, 1986 ; Margules, 1986) ou de la biodiversité (Blondel, 1994). Nous proposons ci-dessous (Tableau 1) un canevas méthodologique qui, sans être exhaustif, peut servir de guide pour évaluer des écosystèmes miniers.

\section{Intervenir pour l'intérêt écologique?}

Des actions récemment conduites "en faveur" de la valeur écologique des sites de carrières, et présentées au Colloque "Recréer la nature", ressortent deux tendances fréquentes :

- il faut diversifier au maximum le site à réaffecter ; il faut accélérer la succession écologique : reverdir les sites au plus vite, boiser, essayer de s'approcher du modèle climax... Nous pensons que ces deux objectifs méritent nuance et discussion.

\section{Diversifier?}

Il est certain que la diversification du biotope joue souvent en faveur de la biodiversité qu'il est susceptible d'accueillir. Mais il faut rappeler les conditions insulaires de bien des sites de carrières : de par leur isolement et leur petite taille, leur richesse spécifique potentielle reste réduite, surtout pour les espèces exigeantes en espace. Dans ces conditions, rien ne sert de diversifier un site trop exigu : on fragmente ses habitats en éléments trop petits pour que s'y installent des populations d'espèces spécialisées. Un objectif plus intéressant pourrait être d'y rechercher la typicité ou l'originalité, en lui assignant un type d'habitat précis (Margules, 1986 ; Usher, 1986a). Lorsque les sites sont nombreux dans une même région, on pourra utilement jouer sur la diversité inter-sites : par exemple, créer une grande roselière sur toute une sablière en eau peut être plus efficace pour la faune qui en dépend que des bouquets de roseaux dispersés sur plusieurs bassins. Cette démarche en faveur d'un maillage d'écosys-

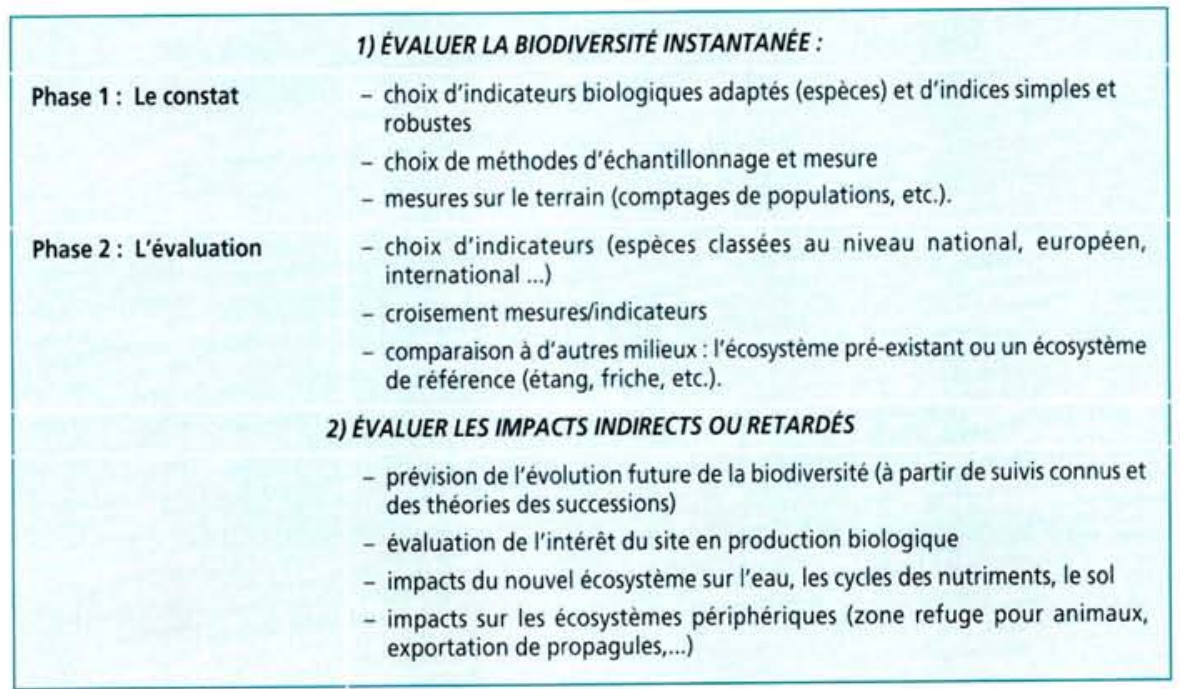

Tableau 1 - Comment apprècier la valeur écologique d'une carrière? 
tèmes représentant les divers stades des successions pourrait s'inspirer de celle proposée par Durrer (1992) pour "renaturer" des anciens bras du Rhin, milieux qui présentent des analogies avec certaines sablières en eau (Tester, 1992). Des sites proches peuvent aussi fonctionner en synergie (effet d'archipel, valable notamment pour les oiseaux : Bournaud et al., 1980, 1982).

\section{Accélérer ou ralentir la succession?}

Ces deux modes d'intervention ne favorisent pas les mêmes espèces. Mais il faut rappeler que les écosystèmes neufs poursuivent, de toute manière, une évolution vers des stades mûrs et que les stades pionniers sont relativement éphémères (et coûteux à entretenir, voire à rétablir, le cas échéant). Dans le cas des carrières, on ne voit donc guère l'intérêt, pour le patrimoine naturel, d'accélérer systématiquement le vieillissement de milieux qui vont évoluer d'eux-mêmes, et parfois rapidement, en ce sens (Arnal et Lamade, 1986 ; Muller, 1994). Le reverdissement artificiel ne doit pas être présenté comme une mesure qui augmenterait automatiquement (Moiroud et Capellano, 1994) l'intérêt floristique ou faunistique des sites.

Par contre, dans le cas des mines, il peut être nécessaire de juguler les phénomènes de pollution organo-métallique par fixation du terrain et reconstitution d'un sol en mettant en place des opérations de revégétalisation artificielle ou de reconstitution de sols par épandage de terre de surface (Daniels et Zipper, 1995 ; Grunwald et al., 1995). Les phénomènes de recolonisation naturelle vont être accélérés dès lors que du sol sera apporté sur les sites miniers (Densmore, 1995).

Enfin, sur les terrils, l'installation d'espèces pionnières fixatrices d'azote atmosphérique (Aulne) ou l'aide à la mycorhization pourront favoriser et accélérer la dynamique de colonisation végétale.

Ce choix de gestion soulève la question importante de l'évolution de la valeur écologique. Dans l'exemple, cité plus haut, des sablières de Quincey, il y a banalisation progressive du peuplement d'oiseaux (figure 4), les espèces ubiquistes étant pratiquement les seules à subsister après une vingtaine d'années. Ne s'agirait-il pas là d'une tendance générale dans les carrières en eau ? (et qui s'accompagne d'une baisse de productivité (figure 3) rappelant celle observée classiquement dans les étangs et réservoirs après quelques années). Nous manquons aujourd'hui d'éléments pour y répondre complètement. L'étude des insectes des carrières sèches en roche massive montre une évolution différente, au moins à l'échelle d'observation du demi-siècle : sur cette durée, l'intérêt écologique, évalué à partir des peuplements entomologiques, ne cesse de croître (G.R.M.R.B., 1983) ; mais le déroulement des successions sur ces substrats compacts et arides est extrêmement lent, de sorte que la végétation y conserve des aspects pionniers plus de cinquante ans après la fin de l'extraction ! Les vitesses d'évolution des successions peuvent donc différer énormément d'un type de carrière à l'autre et, dans tous les cas, la composition floristique et faunistique d'un écosystème évoluant librement se modifie pendant au moins plusieurs siècles. Ainsi, les études botaniques soulignent fréquemment la banalisation progressive des peuplements végétaux qui évoluent vers la forêt sur des sols décapés (Holl et Cairns, 1994), aussi bien sur roche massive ou alluvionnaire que dans le cas particulier des tourbières ; dans ce dernier cas notamment, la reprise d'une exploitation modérée est préconisée pour ramener le milieu à des stades jeunes, pionniers, jugés originaux et intéressants sous l'angle floristique. À titre de comparaison, relevons une tendance qui apparaît nettement à l'analyse des peuplements d'oiseaux (très étudiés) des forêts du Paléarctique : les études menées depuis une trentaine d'années montrent une très forte convergence des peuplements d'origines géographiques diverses au cours du déroulement des successions (Benyacoub, 1993 ; Blondel, 1990 ; Ferry et Frochot, 1990). Ainsi, les peuplements d'oiseaux des stades forestiers pionniers d'Afrique du Nord, de France ou de Pologne sont très différents, tandis que ceux des stades mûrs ne le sont pas, et comportent beaucoup d'espèces en commun. Il en résulte que les espèces de la forêt mûre, qui forment le " fond de l'avifaune du Paléarctique " (Blondel, 1986b, 1990), ont une très large répartition et restent le plus souvent dépourvues d'identité régionale, au contraire des espèces pionnières. Ces dernières seront donc plus facilement classées comme indicatrices d'originalité ou de rareté et contribuent à accroître la valeur écologique selon ces critères.

Lors des entreprises de réaffectation, les interventions doivent donc être nuancées en fonction des réalités écolo. giques. Les coûts des interventions devront aussi être mieux pris en compte et comparés aux résultats obtenus (Point, 1994). Un objectif raisonnable pourrait tout aussi bien être de laisser faire la nature et, quand on intervient, de ne le faire qu'avec des objectifs rigoureux et des connaissances précises.

\section{Voies de recherches}

Dans les sites de carrières et mines se développent donc des écosystèmes originaux, dont la valeur patrimoniale s'avère très variable dans l'espace et dans le temps. Notre connaissance du fonctionnement de ces milieux reste encore très partielle, et suggère des voies de recherches, dont les deux suivantes semblent particulièrement d'actualité.

La première est d'ordre méthodologique : il faut poursuivre les travaux sur l'évaluation des écosystèmes. Le cas des carrières fait ressortir le besoin d'outils d'évaluation et d'écosystèmes de référence (Jefferson et Usher, 1986 ; Robert et al., 1991). Plutôt que de se limiter à développer des méthodes propres aux sites miniers, il serait utile de disposer aussi d'outils largement applicables à tous les écosystèmes, au niveau national ou européen, pour permettre des comparaisons sur des bases scientifiques solides et à des échelles différentes.

Le second thème, celui des successions écologiques, relève d'abord de la recherche fondamentale. Après la pertur- 
bation majeure représentée par l'extraction des roches, les écosystèmes neufs (qu'ils soient abandonnés, réhabilités ou réaffectés) se transforment considérablement. On manque encore de données précises sur ce processus, d'une part parce que très peu de suivis pluriannuels sont réalisés et d'autre part parce que les milieux miniers sont relativement récents, d'où la rareté des modèles permettant d'apprécier leur évolution à long terme (de l'ordre du siècle). Il conviendrait donc de lancer des programmes de recherche sur les successions se développant dans les divers types de carrières et mines :

- par l'approche synchronique : essayer de dégager, par comparaison de sites d'âges différents, les grandes tendances d'évolution de ces écosystèmes ; il faudrait étudier plus spécialement les milieux issus des exploitations les plus anciennes ;

- par l'approche diachronique : mettre en place des suivis, sur des durées les plus longues possible, permettant l'analyse fine de sites définis. Cela suppose d'employer des protocoles assez simples et robustes pour résister à l'usure du temps, de maîtriser suffisamment le site pour éviter les perturbations intempestives et de disposer de garanties pour des financements à long terme...

Ces conditions sont difficiles à réunir, mais le thème des successions justifie de tels programmes, aussi bien pour la connaissance fondamentale des écosystèmes de carrières que pour leur gestion et leur évaluation.

\section{Références}

Amoros C. et Petts G.E. (1993). Hydrosystemes fluviaux, Paris, Éd. Masson.

Arnal G. et Lamade E. (1987). La reconquête par la végétation spontanée des anciennes carrières de sable de Fontainebleau, Bull. liaison Labo. P. et Ch., 152, 9-16.

Aronson I. Floret $C$, Le Floc'h E., Ovalle $C$. et Pontanier $R$. (1993). Restoration and Rehabilitation of degraded Ecosystems in Arid and semi-Arid Lands. I. A View from the South, Restoration Ecology, 8-17.

Barret P. (1994). Territoires dégradès, quelles solutions? Fondation de France, $116 \mathrm{p}$.

Benyacoub S. (1993). Écologie de l'avifaune forestière nicheuse de la région d'El-Kala (Nord-Est algérien). Thèse Université de Bourgogne, Dijon.

Blandin (1986). Bioindicateurs et diagnostic des systèmes écologiques, Bull. Ecologie, 17, $n^{\circ} 4,215-307$

Blondel J. (1976). Stratégies démographiques et successions écologiques, Bull. Soc Zool. France, 101, 695-718.
Blondel J. (1979). Biogéographie et Écologie, Paris, Masson. Blondel J. (1986 a). Biogéographie évolutive, Paris, Masson. Blondel J. (1986 b). Biogéographie évolutive à différentes échelles: I'histoire des avifaunes méditerranéennes. in Ouellet H. éd., Acta $19^{\circ}$ Congr. Int. Ornith., Ottawa, 155-188.

Blondel J. (1990). Biogeography and history of forest bird faunas in the Mediterranean zone, in Keast A. éd. Biogeography and Ecology of Forest Bird Communities, The Hague, SPB Academic Pub., 95-107.

Blondel J. (1994). Les apports de la biologie de la conservation, Communication Colloque "Recréer la nature", cet ouvrage.

Bornette G., Amoros C. et Chessel D. (1994). Effect of allogenic processes on successional rates in former river channels, Journal of Vegetation Science, 5, 237-246.

Bournaud M., Ledant J. P., Broyer J. et Richoux M. (1980). Influence des paramètres physionomiques du milieu étang sur la distribution des oiseaux en période de nidification, Le Bièvre, 2, 25-47.

Bournaud M., Ledant J.P., Broyer J. et Richoux M. (1982). L'espace étang dans ses rapports avec l'avifaune en période de nidification, Bull. Ecol., 13, 125-144.

Brett Y.B. (1994). Et les terrils reverdiront, L'Environnement Magazine, 1524, 48-49.

Brunaud A. (1987). L'évaluation des milieux : comment faire ? pourquoi faire ? Bull. Soc. Hist. nat. Autun, 120, 3-27.

Brunaud A., Cerveaux P. et Bert D. (1988). La végétalisation des milieux handicapés : cas des gravières en eau et de talus routiers, Bull. Soc. Hist. nat. Autun, 125, 3-29.

Cardinal C. (1983). Composition et évolution saisonnière du phytoplancton du lac de Créteil (Val de marne / France). Sciences de l'eau, 2, 153-172.

Carteron M. et Cachot J.L. (1985). Création d'une réserve biologique dans une ballastière de la région de Faverney. Rapport Université de Franche-Comtè, Écologie animale.

Cerveaux P. (1987). Étude des macrophytes (aquatiques et subaquatiques) des gravières en eau : conditions de leur installation et de leur évolution. Rapport DEA Universités Dijon et Lyon I.

Clements F.E. (1916). Plant succession : an analysis of the development of vegetation, Carnegie Inst. Washington Public. 242, 1-512.

Daniels W. L. et Zipper C. E. (1995). Improving coal surface mine reclamation in the Central Appalachian region. in Rehabiliting damaged ecosystems, J. Cairns Jr (ed.), CRC Press Inc.,187-217. Densmore R.V (1994). Succession on regraded placer mine spoil in Alaska, U.S. A., in relation to initial site characteristics, Arctic and Alpine Research, 26, $n^{\circ}$ 4, 354-363.

Desbrosses R. (1982). Premiers stades de la colonisation d'une sablière par la faune aquatique, Rapport DEA Universités Dijon et Lyon 1.

Devillers P., Beudels R.C., Devillers-Terschuren J., Lebrun P. Ledant J.P. et Serusiaux E. (1990). Un projet de surveillance de l'état de l'environnement par bio-indicateurs, Les Naturalistes belges, 71, 3- spécial "Orchidées $n^{\circ}$ 4", 75-98.

Durbec A. (1986). Sectorisation des berges des ballastières en eaux. Application à l'ètude des échanges hydrodynamiques avec la nappe phréatique d'Alsace, au nord de Strasbourg, Thèse Université de Strasbourg, 204 p.

Durrer H. (1992). Stratégie et concept de renaturation d'une partie de l'Obere et de la Mittlere Au en Petite Camargue Alsacienne, In Actes du séminaire Gestion et restauration des milieux fluviaux, Mulhouse, Conf. Permanente des Réserves Naturelles, 48-56.

Eberentz P. et Rinck G. (1987). Impact qualitatif des carrières en eau sur les nappes souterraines, B.R.G.M., Taxe parafiscale sur les granulats, octobre 1987.

Ferry C. (1960). Recherches sur l'écologie des oiseaux forestiers en Bourgogne. I. L'avifaune nidificatrice d'un taillis sous futaie de Querceto-carpinetum scilletosum, Alauda, 28, 2, 93-123. 
Ferry C. et Frochot B. (1970). L'avifaune nidificatrice d'une forêt de chênes pédonculès en Bourgogne. Etude de deux successions écologiques, La Terre et la Vie, 24, 153-251.

Ferry C. and Frochot B. (1990). Bird communities of the forests of Burgundy and the Jura (Eastern France), in Keast A. ed. Biogeography and Ecology of Forest Bird Communities, The Hague, SPB Academic Pub., 183-195.

Frochot B. et Renier J. (1984). Une mise en valeur écologique des sablières en eau. L'exemple de Saule-Guillaume, ministère Environnement et Université de Dijon.

Gambade G. et Simonin-Guengard A.M. (1991). Une gravière bourguignonne d'un type nouveau : le Lac de Chour (Côte d'Or), Bull. sci. Bourg., 44, 1+2, 1-21.

Garnier J. (1982). Production primaire d'une sablière (lac de Créteil, Val-de-Marne). Thèse Université Paris VI.

Garnier J., Lelong J.F. et Meybeck M. (1987). Comparaison physico-chimique et biologique de sept bassins artificiels dans les alluvions de la région parisienne, Naturaliste can. (Rev. Ecol. Syst.), 114, 325-342.

Genin B. (1987). Les populations de Mollusques Gastéropodes des sablières en eau (Côte d'Or). Colonisation, évolution. Rapport DEA Universités Dijon et Lyon I.

Glue D.E. (1970). Changes in the bird community of a Hampshire gravel pit 1963-68, Bird Study, 17, 1, 15-27.

G.R.M.R.B. (1983). Mecanismes fondamentaux de repeuplement par la faune et la flore des milieux artificiels resultant de l'activité industrielle (carrieres, ballastières hors eau), Rapport convention de recherche. Ministère de l'Environnement, Université Besançon, 179 p.

Grunwald C., Iverson L. R. et Szafoni D. B (1995). Abandoned mines in Illinois and North Dakota : toward an understanding of revegetation problems, in Rehabiliting damaged ecosystems, J. Cairns Jr (ed.), CRC Press Inc., 385-411.

Holl D. K. et Cairns J. Jr (1994). Vegetational community development on reclaimed coal surface mines in Virginia, Bulletin of the Torrey Botanical Club, 121 (4), 327-337.

Hughes S.W.M. (1992). Breeding productivity of Little Grebe, British Birds, 85, 10, 555-556.

Jaffre T., Rigault F. et Sarrailh J.M. (1994). Restauration des anciens sites d'exploitation du minerai de nickel en NouvelleCalédonie, poster Colloque "Recreeer la nature".

Jefferson R. et Usher M. (1986). Ecological succession and the evaluation of non-climax communities. in Usher M., Wildlife Conservation Evaluation, London, Chapman and Hall, 69-91.
Jorgenson M. T. et Joyce M. R. (1994). Six strategies for rehabiliting land disturbed by oil development in Arctic Alaska, Arctic, 47, $n^{\circ} 4,374-390$.

Labroue J., Tourenq J.N., Mieussens C., Robert J. et Donville B. (1988). Rôle des lacs de gravières dans la diminution des teneurs en nitrates des aquifères alluviaux de la vallée de la Garonne. Essai de quantification, Annals Limnol., 24 (1), 31-38. Le Floc'h E. et Aronson J. (1994), Écologie de la restauration : réponses à la dégradation des écosystèmes et à la fragmentation des paysages. Concepts, vocabulaire et applications, Communication Colloque "Recréer la nature", cet ouvrage.

Ledant J.P. (1991). Remarques critiques sur le choix des critères d'évaluation biologique, Annales de Gembloux, 97, 157-176.

Lefeuvre J.C. et Le Duc J.P. (1981). La reconquête des milieux naturels : faire revivre les carrières, Annales des Mines, 135-142. Lepart J. et Escarre J. (1983). La succession végétale, mécanismes et modèles: analyse bibliographique, Bull. Écol., 14, 133-178. Margules C. (1986). Conservation evaluation in practice. in Usher M. ed., Wildlife Conservation Evaluation, London, Chapman and Hall, 297-314.

Milne B.S. (1974). Ecological succession and bird life at a new excavated gravel pit, Bird Study, 21, 263-278.

Moiroud A. et Capellano A. (1994). Un procédé de revégétalisation original et peu coúteux de sols dégradés : I'emploi de plantes fixatrices symbiotiques d'azote, poster Colloque "Recreer la nature".

Moreau N. (1982). Étude biogéochimique comparative de différents plans d'eau de la plaine alluviale du Vaudreuil (Eure). Thèse Université d'Orléans.

Muller S. (1994). La restauration des milieux terrestres profitet-elle à la biodiversité ? Communication Colloque "Recreer la nature".

Odum E.P. (1969). The strategy of ecosystem development, Science, 164, 262-270.

Odum E.P. (1993). Ecology and our Endangered Life-Support Systems, Sunderland, Sinauer.

Peaudecerf P. (1975). Effets des gravières sur le comportement hydrodynamique des nappes d'eaux souterraines, $L a$ Houille Blanche, 2/3, 133-140.

Peaudecerf P. (1977). Gravières et prèservation des ressources en eau souterraines, Bull. Inst. Ecol. Appl. Orlèans, 223-229.

Petit D.(1983). Les groupements végétaux colonisateurs des terrils du charbonnage du nord de la France : intérêts de leur étude. in GEHU J.M. éd., Les végétations nitrophiles et anthropogenes, Berlin, Cramer, 155- 178

Point P. (1994). Pour une approche économique de la restauration des écosystèmes, communication Colloque "recréer la nature".

Richardot A. (1983). Influence de l'áge et du marnage des sablières sur quelques groupes faunistiques. Rapport DEA Universités Dijon et Lyon I.

Robert J.C., Cretin J.Y. et Prouteau C. (1991). Typologie des carrieres hors eau de Franche-Comté, Les cahiers de l'environ. nement, Etudes et recherches en ecologie comtoise, 10, 1-32.

Roché J. (1982). Structure de l'avifaune des étangs de la plaine de Saône : influence de la superficie et de la diversité végétale, Alauda, 50, 3, 193-215.

Simberloff D. (1986). Design of nature reserves, in USHER M. éd., Wildlife Conservation Evaluation, London, Chapman and Hall, 315-337.

Sionneau J.M. (1987). Les potentialités écologiques des carrières, Industrie minérale - Mines et carrières, 1-10.

Spain A. et Lavelle P. (1994). Le sol, support de la restauration et de la recréation d'écosystèmes, communication Colloque "Recreer la nature".

Tatoni T., Magnin F., Bonin G. et Vaudour J. (1994). Secondary successions on abandoned cultivation terraces in calcareous Provence. I. Vegetation and soil, Acta Oecologica, 15 (4), 431. 447

Testard P. (1983). Le lac de Créteil (règion parisienne) : un lac de sablière peu profond in Lamotte $M$. et Bourliere $F$. èd. Problèmes d'écologie. Ecosystemes limniques, Paris, Masson, 113-159.

Tester U. (1992), Gestion de la Rèserve Naturelle d'Auried, in Actes du séminaire Gestion et restauration des milieux fluviaux, Mulhouse, Conf. permanente des réserves Naturelles, 57-63.

Usher M. (1986 a). Wildlife conservation evaluation : attributes, criteria and values, in Usher M. èd. Wildlife Conservation Evaluation, London, Chapman and Hall, 3-44.

Usher M. ed.(1986 b). Wildlife Conservation Evaluation, London, Chapman and Hall.

Vermorel P. (1985). Contribution à la connaissance de la sédimentation dans plusieurs plans d'eau artificiels de la plaine alluviale du Val de Reuil (Eure). These Universite d'Orleans.

\section{ABSTRACT : Ecological interest of quarries, gravel pits and coal tips}

Quarries, gravel pits and coal tips happen or not to be spontaneously colonised by flora or fauna. New ecosystems characterized by low biological productivity, original living conditions, isolation and often reduced human presence tend to develop there.

Flora and fauna are often out of the ordinary with species which are rare locally. These ecosystems display a quick evolution following ecological successions in the years after the end of the exploitation.

A twenty year monitoring of the evolution of the bird population of the gravel pits in Burgundy is a good example of such successions. After the end of the exploitation, the list of species, their abundance, their diversity, etc., change quickly from year to year.
The ecological importance of the site which is studied evolves in the parallel way to the number and scarcity of the species : it increases rapidly, then is at its apex around the seventh year, and then decreases progressivly. The bird population tends to be less and less varied and the species more and more common. We may wonder if it corresponds to a general tendancy.

This hypothesis deserves to be studied in other taxa and in other types of sites. An assessment of the available studies concerning quarries or gravel pits ecosystems reveals lacks in scientific research and data : monitoring of the evolution of these ecosystems should be set up as well as methologies permitting a precise evaluation of their ecological interest. 